\title{
Preisfindung von IT-Produkten durch retrograde Kalkulation
}

\section{Einsatz von Quality Function Deployment und modifizier- tem Target Costing für die Preisfindung bei Neu- und Wei- terentwicklungen von IT-Produkten}

\author{
Georg Herzwurm ${ }^{1}$, Benedikt Krams², Wolfram Pietsch² \\ ${ }^{1}$ Lehrstubl für ABWL und Wirtschaftsinformatik II, \\ Betriebswirtschaftliches Institut, Universität Stuttgart \\ ${ }^{2}$ Fachbereich Wirtschaftswissenschaften, Fachbochschule Aachen
}

\section{Einleitung}

Mit dem Übergang von der Individualsoftwareentwicklung auf die Entwicklung von Software-Produkten ändern sich auch die Anforderungen an die Abrechnung der betreffenden Dienstleistungen. Während die Abrechnung von Softwareentwicklungsleistungen zumindest früher zumeist „nach Aufwand“ erfolgte - Preis gleich Aufwand plus Gewinnaufschlag (s. u.) - wird für Standardsoftware wie für andere Produkte ein bestimmter Preis definiert. Die Bestimmung eines (Fest-)Preises ist aufgrund unzuverlässiger Aufwandschätzung in der Praxis auch bei Individualsoftwareentwicklungen eine wichtige Option. Darüber hinaus gibt es immer mehr industriell gefertigte Produkte, deren Wertschöpfung durch Software bestimmt wird und andererseits spezifische Softwaresysteme, die in eine bestimmte Hardwareplattform eingebettet sind. Die Verfahren für die Kalkulation und Preisfindung ${ }^{1}$ industrieller Produkte lassen sich jedoch nicht ohne weiteres auf Software-Produkte übertragen. Eine klare Abgrenzung und Zuordnung funktionaler Elemente aufgrund der unterschiedlichen Technologien ist schwieriger und die Funktionen werden aufgrund der Immaterialität von Software anders bewertet. Dies gilt prinzipiell für alle IT-Produkte, d. h. für softwareintensive Produkte mit oder ohne Hardwareanteile. Bei der Bestimmung des Preises von IT-Produkten

\footnotetext{
${ }^{1}$ In der wissenschaftlichen Literatur werden für die Terminologie Preisfindung Synonyme verwendet. Unter anderem die Begriffe Preisbestimmung, Preisermittlung, Preisfestlegung als auch Preissetzung (z. B. Buxmann et al. 2008, S. 86; Schierenbeck 2003, S.291f.). Im Folgenden wird ausschließlich der Begriff Preisfindung verwendet.
} 
kommt der Identifikation und Priorisierung der relevanten Kundenanforderungen eine Schlüsselrolle zu, und zwar sowohl bei deren Neu- als auch Weiterentwicklung. QFD hat sich als verlässliches Instrument für die Identifikation originärer Kundenanforderungen an IT-Produkte bewährt. Im Folgenden wird diskutiert, ob QFD in Kombination mit retrograder Kalkulation im Sinne des Target Costing für die Preisfindung neuer und weiterzuentwickelnder IT-Produkte eingesetzt werden kann.Kostenrechnungsmethoden für industriell gefertigte Konsumgüter sind methodisch ausgereift und praxiserprobt. So ermöglicht die in der Automobilindustrie etablierte Prozesskostenrechnung eine verursachungsgerechte Aufteilung der Kosten der Entwicklung und der Produktion auf die zu fertigenden Produkte. Eine solche Zuordnung ist für IT-Produkte nicht unmittelbar möglich. Software wird nicht im industriellen Sinne gefertigt, sondern entwickelt und anschließend installiert. Die Wertschöpfungsketten der Erstellung der Leistungen unterscheiden sich grundsätzlich: Das Lizenzgeschäft (ggf. inklusive Services), das Projektgeschäft oder weiterführende Modelle wie das Systemservicegeschäft weisen andere Voraussetzungen, Stufen und Gegenstandbereiche auf (Herzwurm und Pietsch 2009, S. 31ff.).

Die unterschiedliche Wertschöpfung in IT-Unternehmen erfordert eine spezifische Preisfindung. Klassische Ansätze der Preispolitik wie die kosten-, wettbewerbs- und marktorientierte Preispolitik können für IT-Produkte und angrenzende Services übertragen werden; die Zielführung für eine adäquate Bedienung der Kundenbedürfnisse und insbesondere der Preisfindung durch kostenorientierte Preispolitik ist jedoch begrenzt (Buxmann et al. 2008, S. 78).

Während bei der kostenorientierten Preispolitik die entstandenen Kosten um einen Gewinnaufschlag erhöht werden, wird bei wettbewerbsorientierter Politik der Preis durch Beobachtung des Marktes und des Wettbewerbs bestimmt. Bei dem nutzenorientierten Ansatz wird die Frage gestellt, was der Kunde für das Produkt zu bezahlen bereit ist (subjektive Zahlungsbereitschaft: Buxmann et al. 2008, S. 87).

In engem Zusammenhang mit der Preispolitik stehen die Methoden zur Preisfindung. Gängig und weit verbreitet sind progressive Kalkulationsverfahren, die in den Kontext der kostenorientierten Preispolitik eingeordnet werden können. Diese führen zum Verkaufspreis derart, dass auf die entstandenen (tatsächlichen) Kosten ein Gewinnaufschlag addiert wird. Für IT-Produkte ist diese Methode (sowohl für Neu- als auch Weiterentwicklungen) wenig zielführend, da entstandene Kosten selten an den Kunden in gleicher Form weitergereicht werden können.

Ein anderer Ansatz ist, dass für das Produkt Festpreise gesetzt und in den Markt kommuniziert werden. Für IT-Produkte ist diese Herangehensweise fordernd; Entwicklungskosten sind schwierig im Vorfeld zu bestimmen. Aufwandschätzmethoden unterstützen Planer bei dieser Form der Preisfindung, liefern aber nur bedingt verlässliche Aussagen.

Vielversprechender sind Kalkulationsmodelle, die nicht vom Aufwand auf den Preis schließen, sondern umgekehrt bzw. retrograd einen erzielbaren Marktpreis 
zugrunde legen, unabhängig, ob dieser nutzen- oder wettbewerbsorientiert ermittelt wurde. Ausgehend von dieser Preisgrenze, die als oberes Limit für die Entwicklungskosten ${ }^{2}$ gesehen werden muss, werden die Kosten auf einzelne Produktfunktionen herunter gebrochen.

Für die Identifizierung der relevanten Produktfunktionen bietet QFD die Methodik, Kundenanforderungen zunächst zu identifizieren, und im Anschluss in Abhängigkeit der Wesentlichkeit für die Befriedigung der Kundenbedürfnisse zu priorisieren. Modifiziertes Target Costing ermöglicht bei Produktneuentwicklungen die Überprüfung, ob die Produktbestandteile innerhalb der zulässigen Produktentwicklungskosten liegen; bei Produktweiterentwicklungen ermöglicht Target Costing Zielvorgaben für weitere Kostensenkungen.

\section{QFD und Target Costing für IT-Produkte}

Im Gegensatz zu vielen einschlägigen Methoden der Qualitätssicherung zeichnet sich QFD dadurch aus, dass nicht alle technisch möglichen Anforderungen berücksichtigt werden, sondern der Fokus auf die vom Kunden geforderten Anforderungen gelegt wird. Für eine zielkostenkonforme Produktentwicklung ist eine effektive Priorisierung der Anforderungen unerlässlich. Ziel sind nicht technische Höchstleistungen und Perfektion, sondern möglichst gut verkäufliche Produkte (Ehrlenspiel 2003, S. 616).

Target Costing ist ein Managementansatz, mit dem frühzeitig und umfassend eine marktorientierte Gestaltung von Produkten und Dienstleistungen sichergestellt werden soll (Arnaout 2001, S. 32). QFD und Target Costing können wie im Folgenden dargestellt für die Entwicklung von Standard- und Individualsoftware einzeln als auch kombiniert eingesetzt werden. Die Relevanz für ITProduktentwicklungen wird in Kapitel 3 für Produktneu- und Weiterentwicklungen diskutiert.

\subsection{QFD}

Voraussetzung für QFD zur kundenorientierten Produktentwicklung ist die Informationsbeschaffung in Form von eindeutigen Anforderungen von den Stakeholdern. Der Anforderungskatalog als Ergebnis der Informationsbeschaffung geht in das sogenannte House of Quality (HoQ), eine zentrale Bewertungsmatrix im Rahmen von QFD, als Kundenanforderungen ein. Kundenanforderungen werden den Produktcharakteristika in einer Matrix gegenübergestellt. Produktcharakteristika liefern die Antwort auf die Frage, wie eine Anforderung umgesetzt werden kann.

\footnotetext{
2 In der Praxis werden auch Entwicklungsbudgets von Entscheidern vorgegeben. Für die Bestimmung dieser Budgets können retrograde Kalkulationsmethoden ebenfalls herangezogen werden.
} 


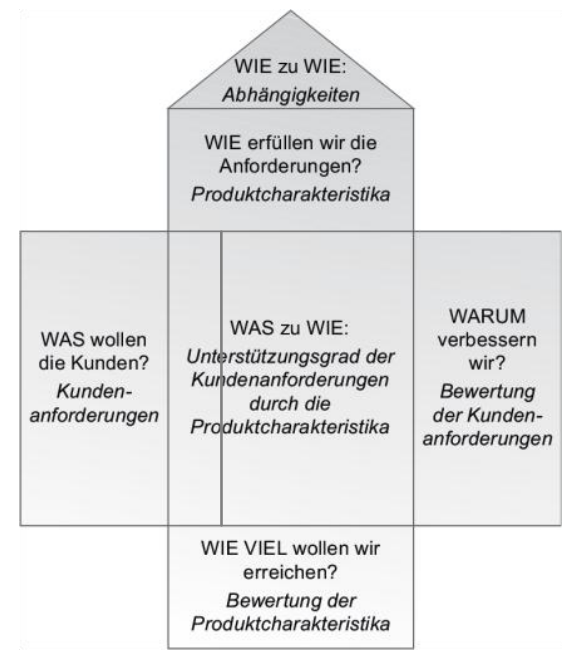

Abbildung 1: Schematische Darstellung des HoQ (in Anlehnung an (Cohen 1995, S. 12))

„Die grundsätzliche Idee ist, gewisse vorgegebene Anforderungen (WAS) den Möglichkeiten zur Anforderungserfüllung (WIE) gegenüberzustellen (WAS zu WIE). Konkrete Angaben zur Existenz der Anforderungen (WARUM), die vorhandenen Anhängigkeiten der Lösungsmöglichkeiten untereinander (WIE zu WIE) und die konkreten Entwicklungsvorhaben (WIE VIEL) komplettieren das Haus“ (Herzwurm und Pietsch 2009, S. 162).

Die Zellen in der Matrix der aufeinandertreffenden Anforderungen und Charakteristika (WAS zu WIE) werden mit einer Skala bewertet, die den Grad der Erfüllung einer Anforderung durch ein Produktmerkmal beschreibt. Die Korrelation reicht dabei von stark (Bewertung erfolgt durch eine 9) über mittel (3 Punkte) und schwach (1 Punkt) bis hin zu keiner Korrelation (0 Punkte) (Saatweber 1997, S. 37).

Das HoQ stellt den Kern von QFD dar, ist jedoch nicht alleiniger Bestandteil. In wissenschaftlicher Literatur und Praxis werden weitere „Häuser“ beschrieben, die aus dem ersten HoQ entwickelt werden. Diese Matrizen dienen bspw. in einem zweiten Schritt der Komponenten- oder Einzelteilplanung.

Weitere Schritte sind die Prozessplanungsphase sowie die Produkt- bzw. Fertigungsplanung, die in der Praxis jedoch selten durchgeführt werden (Vier-PhasenModell: (Saatweber 1997, S. 40f.)).

Die Vorgehensweise des Vier-Phasen-Modells stellt eine Variante von QFD dar, und ist für die Entwicklung von Serienprodukten vorgesehen (Gustafsson 1996, S. 25). Für den Einsatz in Softwareentwicklungsprojekten müssen Modifikationen der Vorgehensweise vorgenommen werden, wie etwa die Erweiterung des HoQ um weitere Ebenen und Matrizen (bspw. Baumöl 1999, S. 215f.; Herzwurm et al. 1995, S. 2f.; Lämmle 2003, S. 104). Zu der Erweiterung zählen bspw. intensivierte Stakeholderanalysen (Customer-Deployment) und Voice-of-Engineer- 
Tabellen zur Analyse der Anforderungen bei der Umsetzung seitens der ITProduktentwickler (Lämmle 2003, S. 145) oder Qualitätstabellen (Baumöl 1999, S. 218f.).

\subsection{Target Costing}

Durch Target Costing werden „Kostenstrukturen frühzeitig im Hinblick auf die Marktanforderungen“ (Horváth 1993, S. 4) gestaltet. Bei diesem marktorientierten Ansatz handelt es sich, im Gegensatz zu ingenieur- und produktfunktionsorientierten Ansätzen, um eine die marktgerechte Produktgestaltung hervorhebende Sichtweise (Arnaout 2001, S. 32ff.).

Target Costing ist ein retrogrades Kalkulationsverfahren, dass im Gegensatz zu progressiven Verfahren dadurch gekennzeichnet ist, dass die Kalkulation eines Produkts rückwärts erfolgt, der Endpreis nicht durch die Summe der in das Produkt einfließenden Kosten zuzüglich Gewinnaufschlag determiniert wird, sondern durch eine holistische Ansatzweise, der der erzielbare Preis auf dem Käufermarkt zugrunde liegt: Vom erzielbaren Marktpreis wird die Gewinnmarge abgezogen um die Zielkosten zu erhalten.

Die Zielkosten werden auf die Produktbestandteile herunter gebrochen und gelten so als qualifizierte Vorgabe für die Entwicklung. Durch Gegenüberstellung des tatsächlichen Kostenanteils eines Produktbestandteils mit der gewichteten Bedeutung eines Produktbestandteils lassen sich teilbezogene Indizes in einem Zielkostendiagramm abtragen.

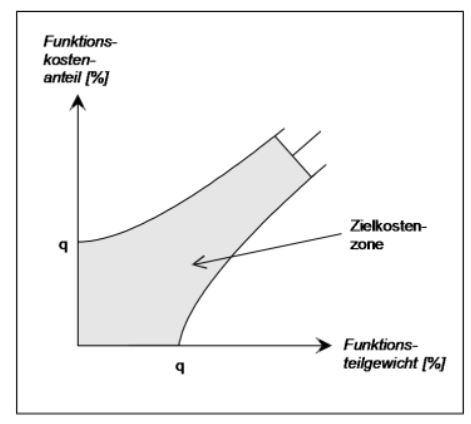

Abbildung 2: Zielkostenkontrolldiagramm (in Anlehnung an Arnaout (2001, S. 53))

Die Zielkostenzone gibt den Bereich an, in dem die Produktbestandteile erfasst sind, deren Verhältnis von Bedeutung (Funktionsteilgewicht) und Kostenanteil (Funktionskostenanteil) akzeptiert wird.

Liegen Produktbestandteile außerhalb der Zielkostenzone bieten sie Potential zur Kostenreduktion (Werte liegen Oberhalb der Winkelhalbierenden), oder es muss die Notwendigkeit der Wertsteigerung geprüft werden (Werte liegen unterhalb der Winkelhalbierenden) (Mayer 2000, S. 85). Somit werden außerhalb der Zielkostenzone Produktfunktionen identifiziert, deren Erstellung von Kunden 
nicht toleriert werden (,zu teuer“, „zu aufwändig“), oder aber deren Erstellung höher monetarisiert werden kann (,zu billig“, „zu einfach“). Beispielsweise kann die Funktionalität eines Servers für eine On-Demand-Lösung ein hohes Funktionsteilgewicht (Kunde gewichtet diese Funktionalität hoch, da ohne Server die Anwendung nicht läuft) bei sehr niedrigem Funktionskostenanteil (schnelle Amortisation nach der Anschaffung) besitzen; der Server ist dann unterhalb der Winkelhalbierenden positioniert.

Das Zielkostendiagramm identifiziert somit Produktbestandteile, deren Bedeutung vom Anbieter falsch eingeschätzt wurden ${ }^{3}$ (am Beispiel der On-DemandLösung: Die Funktionalität des Servers könnte „teurer verkauft“ werden).

\section{Einsatz von QFD und modifiziertem Target Costing für die Preisfindung von IT-Produkten}

Die gemeinsame Anwendung von QFD und Target Costing ist in der wissenschaftlichen Literatur bereits diskutiert (z. B. Baumöl 1999, S. 216; Ehrlenspiel 2003, S. 217; Lämmle 2003, S. 168; Mayer 2000, S. 115; Saatweber 2007, S. 464). Durch Kombination beider Methoden werden denjenigen Anforderungen der größten Aufwand zugeordnet, die den höchsten Anteil an der Erhöhung des Kundennutzens und der Befriedigung der Kundenbedürfnisse haben. Die Ergänzung durch Target Costing untersucht in einem zweiten Schritt, ob die priorisiert ausgewählten Anforderungen mit den Zielkosten in Einklang zu bringen sind.

Für IT-Produkte gilt, dass sowohl Neu- als auch Weiterentwicklungen je eine Entwicklungsleistung darstellen. Vereinfachend wird angenommen, dass Neuentwicklungen für Standard- als auch Individualsoftware keinen wesentlichen Unterschieden unterliegen. Für Weiterentwicklungen von Standard- und Individualentwicklungen wird vereinfachend angenommen, dass es sich bei beiden Formen von IT-Produkten um Wartungsaufwände handelt. Begründet liegt diese Annahme darin, dass die für Weiterentwicklungen vorgenommen Modifikationen häufig eine Verbesserung des ursprünglichen Systems darstellen (bspw. ein neues Release zum Schließen etwaiger Sicherheitslücken der Vorgängerversion, etwa bei einem Browser): Es entsteht in der Regel kein neues und/oder innovatives Produkt.

\subsection{Preisfindung für IT-Produkt-Neuentwicklungen}

Wie in Kapitel 1 erwähnt, sollten zur Preisfindung von IT-Produkten die Ansätze der wettbewerbs- bzw. nutzenorientierten Preispolitik übernommen werden. Das hat zur Folge, dass durch Marktanalyse oder Einschätzung des Kaufverhaltens der Kunden der Preis des zu entwickelnden Produkts bereits bestimmt ist.

\footnotetext{
3 Anmerkung: Ein Aufwiegen von Produktbestandteilen, die je oberhalb der Zielkostenzone liegen mit denen, die unterhalb der Zielkostenzone liegen, ist im Target Costing nicht vorgesehen.
} 
Sind die Anforderungen identifiziert und mit den Produktfunktionen durch QFD bewertet, erfolgt die Übertragung (in folgender Abbildung durch mittigen Pfeil symbolisiert) der priorisierten Funktionen in das Zielkostendiagramm.

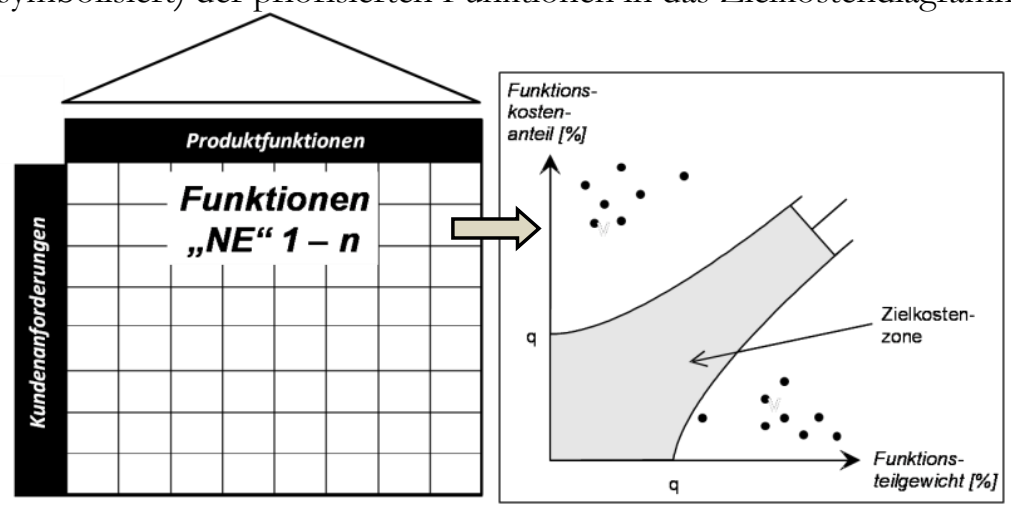

Abbildung 3: Schematisches HoQ für die Neuentwicklung (NE) von IT-

Produkten und Darstellung von Entwicklungskomponenten außerhalb der Zielkostenzone

Bei Neuentwicklungen von IT-Produkten kommt es aufgrund geringer Erfahrung der Entwickler bezüglich des neuen Produkts dazu, dass eine Vielzahl der übertragenen Funktionen außerhalb der Zielkostenzone liegen (s. Abbildung 3: Diese zeigt im Zielkostendiagramm nur die Funktionen außerhalb der Zielkostenzone). Dies gilt insbesondere für Neuentwicklungen von Individualsoftware: Durch die Einmaligkeit der Entwicklung kommt es in der Regel nicht zu einer Nachkalkulation des Produkts (Ehrlenspiel 2003, S. 592)4, auch deshalb, weil das Produkt dem Kunden bereits übergeben wurde. Eine Weiterentwicklung des Produkts zur Senkung der Kosten findet nicht statt.

Bezogen auf die Preisfindung von IT-Produkten für Neuentwicklungen muss festgehalten werden, dass QFD und modifiziertes Target Costing dazu beitragen, für die priorisierten Funktionen die Zielkosten zu bestimmen. Deren Einhaltung wird jedoch in laufenden Entwicklungsprojekten gesteuert und ggf. zu Lasten der geforderten Qualität, Funktionalität oder Zeit erreicht (Teufelsquadrat: Sneed 1987, S. 42; erweitertes magisches Fünfeck: Heilmann 2000, S. 20). Die Findung eines „Zweiten“ Preises (nach Durchführung von QFD und modifiziertem Target Costing, s. a Kapitel 3.2) findet bei Neuentwicklungen somit nicht statt.

\subsection{Preisfindung für IT-Produkt-Weiterentwicklungen}

Ausgangslage für die Preisfindung bei Weiterentwicklungen von IT-Produkten sind die Ergebnisse der bereits für die Neuentwicklung angewendeten Methoden QFD

\footnotetext{
${ }_{4}$ Ehrlenspiel differenziert im Kontext des zielkostengesteuerten Konstruierens zwischen Serien-, Einzel- und Kleinserienfertigung. Seine Aussage bzgl. Einzelfertigung wurde auf Individualsoftwareentwicklungen übertragen.
} 
und modifiziertes Target Costing. Das Zielkostendiagramm der Neuentwicklung (s. Abbildung 3) liefert den Start für Weiterentwicklungsbemühungen als auch das Ziel: Fokussiert werden die Funktionen betrachtet, die außerhalb der Zielkostenzone liegen (Start); Ziel ist, diese Funktionen innerhalb der Zielkostenzone zu platzieren.

Abbildung 4 zeigt das HoQ der Neuentwicklung ergänzt um die Matrix für die Weiterentwicklung des gleichen IT-Produkts sowie das Zielkostendiagramm der Weiterentwicklung.

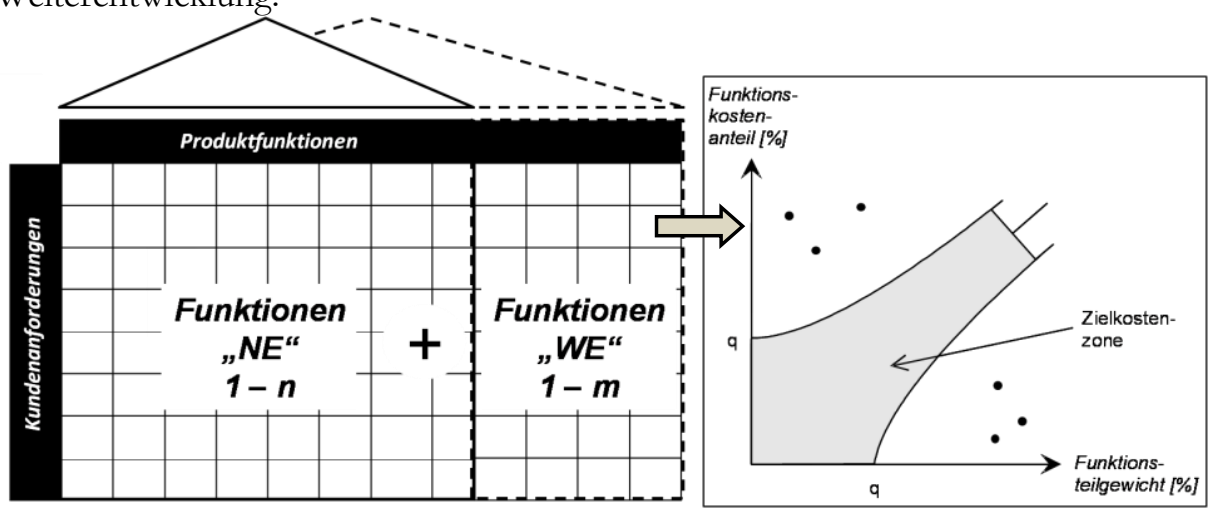

Abbildung 4: Schematisches HoQ der Neuentwicklung ergänzt um die Matrix der Weiterentwicklung (WE) von IT-Produkten und Darstellung von Entwicklungskomponenten außerhalb der Zielkostenzone

Die Abbildung verdeutlicht den Aufbau des HoQ der Weiterentwicklung auf dem der Neuentwicklung. Darüber hinaus zeigt das Zielkostendiagramm gegenüber dem der Abbildung 3 weniger Produktfunktionen außerhalb der Zielkostenzone.

Das liegt darin begründet, dass durch Weiterentwicklung Kosten erneut gesenkt werden: Dies geschieht zum einen bspw. durch schnellere Entwicklungsverfahren oder Substitution eingesetzter Materialien (bei gleichbleibender Qualität). Zum anderen können funktionsseitig Anpassungen derart vorgenommen werden, dass z. B. von Kunden nicht angenommene Funktionen nicht weiterentwickelt werden bzw. Over-Engineering ${ }^{5}$ vermieden wird. ${ }^{6}$

Kostensenkungspotentiale gibt es insbesondere bei der Weiterentwicklung von Standardsoftware: Bei marktseitig geforderten kurzen Entwicklungszeiten und rascher Technologieentwicklung ist die Bedeutung der „Treffsicherheit“ der Kundenanforderungen immer wichtiger (Mayer 2000, S. 115)7. Durch QFD und modi-

\footnotetext{
5 Over-Engineering muss ceteris paribus auch bei Neuentwicklungen vermeiden werden. QFD als auch Target Costing sind ohnehin geeignete Instrumente zur Vermeidung von Over-Engineering (Saatweber 1997, S. 27, S. 253).

${ }^{6}$ Durch die Wiederverwendung der Ergebnisse der Neuentwicklung treten ferner Synergieeffekte auf, die eine Aufwandsreduzierung bei der Durchführung der Methoden QFD und Target Costing zur Folge haben. Diese wirken sich jedoch nicht auf das Zielkostendiagramm aus.

7 Mayer beschreibt den Nutzen von QFD für die Elektronikindustrie bei Serienproduktion. Seine Aussage wurde auf IT-Standardprodukte übertragen.
} 
fiziertes Target Costing und die bereits angestellten Vorüberlegungen zur Neuentwicklung werden im Rahmen der Weiterentwicklung die relevanten Produktfunktionen identifiziert und gezielt verbessert. Für die Preisfindung bei IT-ProduktWeiterentwicklung ergeben sich folgende Konsequenzen:

Durch die Weiterentwicklung ergeben sich Kostensenkungspotentiale, die die Findung eines „zweiten“ Preises ermöglichen (vgl. Kapitel 3.1). Die gesunkenen Entwicklungskosten können entweder an Kunden weitergegeben werden, um einen Vorteil gegenüber dem Wettbewerb zu erzielen (die Gewinnmarge bleibt gegenüber der Neuentwicklung identisch), oder aber gesunkene Entwicklungskosten werden bei gleichem Preis der ursprünglichen Neuentwicklung nicht in den Markt kommuniziert (Erhöhung der Gewinnmarge).

Darüber hinaus kann bei entsprechender Wettbewerbslage ein höherer Preis veranschlagt werden, der darin begründet ist, dass die Funktionalität des ITProdukts verbessert wurde.

\section{$4 \quad$ Fazit}

QFD und Target Costing unterstützen die anforderungs- und marktgerechte sowie zielkostenkonforme Entwicklung von IT-Produkten. Die retrograde Kalkulation durch Kombination von QFD und Target Costing unterstützt eine wettbewerbsund nutzenorientierten Preispolitik und ist für IT-Produkte besser als progressive Kalkulationsverfahren oder die kostenorientierte Preispolitik geeignet.

Für Standard- als auch Individualsoftwareentwicklungen ist die Kombination aus QFD und modifiziertem Target Costing für IT-Produkt-Weiterentwicklungen sinnvoll, weniger jedoch für IT-Produkt-Neuentwicklungen.

Die Kombination von QFD und modifiziertem Target Costing bietet eine Entscheidungsgrundlage für eine Anhebung oder Senkung des Angebotspreises. Ausschlaggebend für die Gestaltung des Angebotspreises ist der „zweite“ Preis (vgl. Kapitel 3.1 und 3.2).

Für IT-Produkt-Neuentwicklungen führt die hier vorgestellte Vorgehensweise nicht zu einer Preisfindung. Das bedeutet nicht, dass die Kombination von QFD und Target Costing unzweckmäßig ist. Vielmehr findet bei Neuentwicklungen unter Anwendung von QFD und Target Costing die Preisfindung für die Zielkostenbestimmung bereits im Vorfeld statt (unter Anwendung der Prinzipien der kosten-, wettbewerbs- und der nutzenorientierten Preispolitik). Darüber hinaus können die Ergebnisse von QFD und Target Costing bei Neuentwicklungen für Nachkalkulationen und das Controlling von Entwicklungsprojekten genutzt werden. 


\section{Literatur}

Akao Y, Mazur GH (2003) The leading edge in QFD: past, present and future. International Journal of Quality and Reliability Management 20 (1): 20 - 35.

Arnaout A (2001) Target Costing in der deutschen Unternehmenspraxis. Eine empirische Untersuchung. In: Horváth P, Reichmann T (Hrsg.) Controlling Praxis. Vahlen, München.

Baumöl U (1999) Target Costing bei der Softwareentwicklung. Eine ControllingKonzeption und instrumentelle Umsetzung für die Anwendungssoftware. In: Horváth P, Reichmann T (Hrsg.) Controlling Praxis. Vahlen, München.

Buxmann P, Lehmann S, Hess T, Staritz M (2008) Entwicklung und Implementierung von Preisstrategien für die Softwareindustrie. In: Gronau N, Eggert S (Hrsg.) Beratung, Service und Vertrieb für ERP-Anbieter. Gito, Berlin.

Cohen L (1995) Quality Function Deployment. How to make QFD Work for You. Prentice Hall, Reading.

Ehrlenspiel K (2003) Integrierte Produktentwicklung. Denkabläufe Methodeneinsatz Zusammenarbeit. Hanser, München.

Gustafsson A (1996) Customer Focused Product Development by Conjoint Analysis and QFD. In: Linköping Studies in Science and Technology, Division of Quality Technology, Department of Mechanical Engineering, Linköping.

Heilmann H (2000) Erfolgsfaktoren des IT-Projektmanagements. In: Etzel HJ, Heilmann H, Richter R (Hrsg.) IT-Projektmanagement, Fallstricke und Erfolgsfaktoren. dpunkt, Heidelberg.

Herzwurm G, Mellis W, Schockert S (1995) Kundenorientierte Planung von Softwareprodukten und -prozessen mit Quality Function Deployment (QFD). In: Oberweis A (Hrsg.): Proceedings Fachgruppentreffen 1995 der GI Fachgruppe 2.5.2 EMISA Entwicklungsmethoden für Informationssysteme und deren Anwendung Requirements Engineering für Informationssysteme, Karlsruhe.

Herzwurm G, Pietsch W (2009) Management von IT-Produkten. Geschäftsmodelle, Leitlinien und Werkzeugkasten für softwareintensive Systeme und Dienstleistungen. dpunkt, Heidelberg.

Horváth P (1993) Target Costing. Schäffer Poeschel, Stuttgart.

Lämmle G (2003) Möglichkeiten der Anwendung von Quality Function Deployment und Zielkostenmanagement am Beispiel von E-BusinessProjekten. Der Andere Verlag, Osnabrück. 
Mayer HJ (2000) Kostenoptimale Prozeßplanung für die mikrotechnische Produktion. In: Fortschritt-Berichte VDI, Reihe 2 Fertigungstechnik, Nr. 536, VDI, Düsseldorf.

Saatweber J (1997) Kundenorientierung durch Quality Function Deployment.

Systematisches Entwickeln von Produkten und Dienstleistungen. Hanser, München.

Saatweber J (2007) Kundenorientierung durch Quality Function Deployment.

Systematisches Entwickeln von Produkten und Dienstleistungen. Symposium Publishing, Düsseldorf.

Schierenbeck H (2003) Grundzüge der Betriebswirtschaftslehre. Oldenbourg, München.

Sneed H (1987) Softwaremanagement. Verlagsgesellschaft Rudolf Müller, Köln. 